\title{
The significance of three-dimensional transesophageal echocardiography assessment for aortic wall papillary fibroelastoma
}

\author{
Toshimitsu Tsugu ${ }^{1,2,3}$ (C) Yuji Nagatomo ${ }^{4} \cdot \operatorname{Jin}_{\mathrm{Endo}^{3}} \cdot$ Masataka Yamazaki $^{5} \cdot$ Hideyuki Shimizu $^{5} \cdot$ Hideo Mitamura $^{2}$. \\ Patrizio Lancellotti ${ }^{1}$
}

Received: 11 March 2019 / Revised: 5 June 2019 / Accepted: 11 June 2019

(c) Japanese Society of Echocardiography 2019

\section{Case}

A 60-year-old woman was referred to our hospital to evaluate hypertension. She had no history of cardiac disease. Transthoracic echocardiography showed mobile low-echoic restiform mass on the aortic side of the aortic valve (AV). It showed no remarkable changes after 1 week of anticoagulation therapy using heparin. Two-dimensional transesophageal echocardiography (2D-TEE) revealed mass had short pedicle which appeared to adhere to the AV commissure between the right and left coronary cusps (Fig. 1a, b). Threedimensional TEE (3D-TEE) showed an $11 \times 9 \mathrm{~mm}$ mobile mass attached to the aortic wall, not AV (Fig. 1c-f). Whole body computed tomography revealed no evidence of infarct area in any organs. Surgical extirpation by minimally invasive procedure through a mini-right thoracotomy was undergone (Fig. 1g-i). Papillary fibroelastoma originated from the aortic wall. Histological findings showed multiple papillary fonds which consisted of acellular matrix by a single layer of endothelial cells, leading to a definite diagnosis of papillary fibroelastoma (Fig. 1k, 1). She had an uneventful course and no recurrence until 6 months after operation.

Toshimitsu Tsugu

tsugu.z7@keio.jp

1 Department of Cardiology, University of Liège Hospital, CHU Sart Tilman, Domaine Universitaire du Sart Tiilman, B.35, 4000 Liège 1, Belgium

2 Department of Cardiology, Federation of National Public Service Personnel Mutual Aid Association Tachikawa Hospital, Tachikawa 190-8531, Japan

3 Department of Cardiology, School of Medicine, Keio University, Tokyo 160-8582, Japan

4 Department of Cardiology, National Defense Medical College Hospital, Tokorozawa 359-8513, Japan

5 Department of Cardiovascular Surgery, School of Medicine, Keio University, Tokyo 160-8582, Japan

\section{Discussion}

Papillary fibroelastoma is the second most common benign cardiac tumor and $77 \%$ of papillary fibroelastoma originates from the valvular endocardium. The non-valvular origin is rare, and only 2 cases of papillary fibroelastoma arising from the aortic wall have been reported [1,2]. Ninety-five percent of papillary fibroelastoma is located in the left heart and the frequency of embolism is as high as 54\% [3]. Complete tumor resection with shave excision yields a high success rate. Therefore, if tumor in the left heart has a mobility, or its size is more than $10 \mathrm{~mm}$, such cases are candidate for surgical resection. According to the retrospective review of 88 patients who were treated surgically for papillary fibroelastoma at Mayo Medical Center [3], the operative procedures for papillary fibroelastoma on $\mathrm{AV}$ were as follows, $83 \%$ for shave excision, $9 \%$ for excision with valve repair, and $6 \%$ for valve replacement. But all cases of papillary fibroelastoma on the aortic wall underwent shaved excision and postoperative course was uneventful without recurrences $[1,2]$. Since the operative procedure may depend on its location, preoperative local diagnosis is crucial. In our case, 3D-TEE correctly detected aortic wall originated papillary 

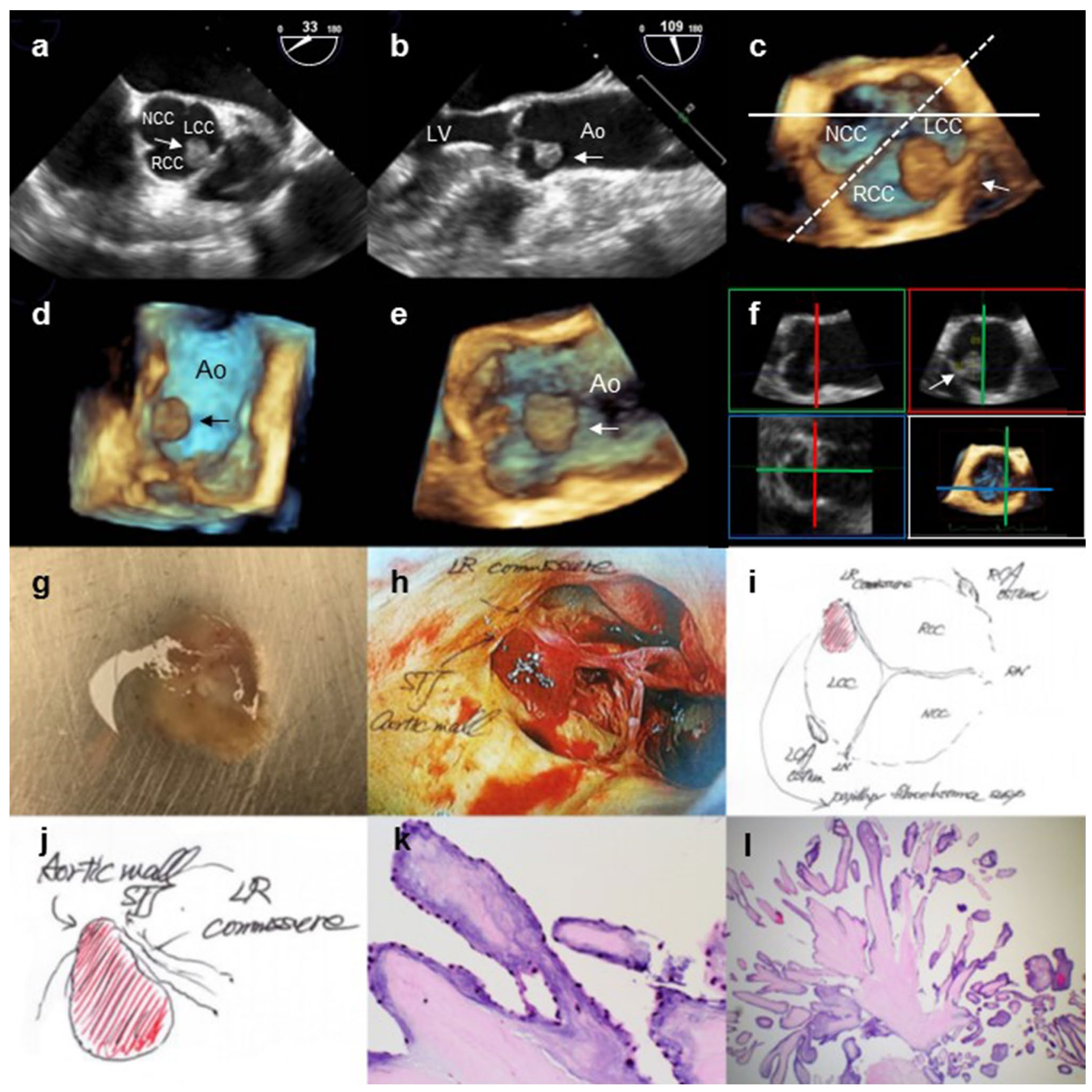
४Fig. 1 a Two-dimensional transesophageal echocardiograms. Midesophageal short axis view ( $33^{\circ}$ rotation) shows a mobile low-echoic restiform lesion (arrow) at the cross section of the aortic valve across the right and left coronary cusps. b Two-dimensional transesophageal echocardiograms. Midesophageal long axis view (109 ${ }^{\circ}$ rotation) shows a mass (arrow) seems to be continuity with the aortic valve and attaches indirectly to the aortic wall. c Three-dimensional transesophageal echocardiograms. The image is cropped at the level of sinus valsalva perpendicular to the ascending aorta. The mass (arrow) has short pedicle and originates from between right and left coronary cusps. d Three-dimensional transesophageal echocardiograms. The image is cropped from cross section of the ascending aorta across the left and non-coronary cusps (c: solid line). The mass (arrow) is attached to the sinus of valsalva and not continuous with the aortic leaflets. e Three-dimensional transesophageal echocardiograms. The image is cropped from cross section of the ascending aorta across the left and right coronary cusps (c: dotted line). The mass (arrow) is separated from the aortic leaflets. f Full volume images are obtained from three-dimensional transesophageal echocardiograms (bottom right). The green (top left) and the blue (bottom left) planes are horizontal to the ascending aorta and vertical to the aortic valve. The red plane (top right) is cross-section of the ascending aorta. The mass (arrow) is directly attached to the aortic wall in cross section of the ascending aorta across the sinus of valsalva (top right). g Resected specimen shows villous and sea anemone such as appearance $(12 \times 10 \mathrm{~mm})$. h Intraoperative photograph. The mass has short pedicle and directly adheres to the aortic wall near the AV commissure between the right and left coronary cusps. $\mathbf{i}$, j Surgical records. The mass directly originated from sinotubular junction in the vicinity of the AV commissure between the right and left coronary cusps. k Hematoxylin and eosin staining shows elongated and branching papillary fronds at low magnification. 1 Hematoxylin and eosin staining shows avascular branching papillary collagen and surrounded by lined flattened endothelial cells at high magnification. $R C C$ rightcoronary cusp, $L C C$ left-coronary cusp, $N C C$ non-coronary cusp, $L V$ left ventricle, Ao aorta fibroelastoma, which mimicked AV origin by 2D-TEE. The assessment by 3D-TEE is a useful approach for the accurate identification of origin of papillary fibroelastoma.

\section{Compliance with ethical standards}

Conflict of interest Toshimitsu Tsugu, Yuji Nagatomo, Jin Endo, Masataka Yamazaki, Hideyuki Shimizu, Hideo Mitamura, and Patrizio Lancellotti declare that they have no conflict of interest.

Ethical standard All procedures followed were in accordance with the ethical standards of the responsible committee on human experimentation (institutional and national) and with the Helsinki Declaration of 1964 and later versions.

Informed consent Informed consent was obtained from all patients for being included in the study.

\section{References}

1. Yerebakan C, Liebold A, Steinhoff G, et al. Papillary fibroelastoma of the aortic wall with partial occlusion of the right coronary ostium. Ann Thorac Surg. 2009;87:1953-4.

2. Gonzalez-Santos JM, Arnaiz-Garcia ME, Vargas-Fajardo Mdel $\mathrm{C}$, et al. Aortic wall papillary fibroelastoma. J Thorac Cardiovasc Surg. 2013;146:e1-3.

3. Ngaage DL, Mullany CJ, Daly RC, et al. Surgical treatment of cardiac papillary fibroelastoma: a single center experience with eighty-eight patients. Ann Thorac Surg. 2005;80:1712-8.

Publisher's Note Springer Nature remains neutral with regard to jurisdictional claims in published maps and institutional affiliations. 